\title{
Tratamento de águas contaminadas por diesel/biodiesel utilizando processo Fenton
}

\section{Treatment of water contaminated by diesel/biodiesel using Fenton process}

\author{
Teofani Koslides Mitre \\ Engenheira Química. Mestre em Engenharia de Minas e Metalurgia. Doutoranda em Saneamento, Meio Ambiente e Recursos Hídricos pelo Departamento \\ de Engenharia Sanitária e Ambiental da Universidade Federal de Minas Gerais (UFMG) - Belo Horizonte (MG), Brasil.
}

\begin{abstract}
Mônica Maria Diniz Leão
Engenheira Química. Doutora em Engenharia Ambiental. Professora Associada do Departamento de Engenharia Sanitária e Ambiental da UFMG -

Belo Horizonte (MG), Brasil.
\end{abstract}

Marcella Cristina Neves Alvarenga

Graduanda em Engenharia Química pela UFMG - Belo Horizonte (MG), Brasil.

\section{Resumo}

A contaminação de águas por misturas diesel/biodiesel pode causar grandes impactos ambientais, relacionados à presença de compostos orgânicos recalcitrantes e tóxicos, inviabilizando o uso de processos biológicos de tratamento. A avaliação da biodegradabilidade, nas proporções B0, B25, B50, B75 e B100 (os números especificam o percentual em massa de biodiesel na mistura), indicou que a adição de biodiesel em teores acima de 50\% aumenta a biodegradabilidade, alcançando 60 e $80 \%$ para B50 e B75, respectivamente. Na aplicação do processo Fenton, a remoção da matéria orgânica foi superior a $80 \%$ em todas as misturas, exceto para B0, que apresentou remoção máxima de 50\%. A oxidação por Fenton se ajustou a um modelo cinético de pseudosegunda ordem em relação à concentração de matéria orgânica, e resultou em aumento da biodegradabilidade de até 150\%.

Palavras-chave: biodegradabilidade aeróbia; biodiesel; cinética de oxidação; reagente de Fenton; processos oxidativos avançados.

\section{Abstract}

Waters contaminated with diesel/biodiesel and their blends can cause major environmental impacts, due to the presence of toxic and recalcitrant organic compounds, which invalidate the use of biological treatment processes. Evaluation of biodegradability of the blends B0, B25, B50, B75 and B100 (the numbers specify the mass percentage of biodiesel in the blend) indicated that the addition of biodiesel at concentrations above $50 \%$ increased biodegradation, reaching 60 and $80 \%$ for B50 and B75, respectively. When the Fenton process was used, removal of organic matter was greater than $80 \%$ in all blends, except for B0, which showed maximum removal of $60 \%$. Oxidation by Fenton was fitted with a pseudo-second order kinetic model in relation to the concentration of organic matter and resulted in increased biodegradation of up to $150 \%$.

Keywords: aerobic biodegradation; biodiesel; kinetics of oxidation; Fenton's reagent; advanced oxidation processes.

\section{Introdução}

Atualmente existe um crescente interesse por fontes alternativas de energia para substituição do petróleo, que é uma fonte limitada e com previsão de esgotamento no futuro. Além de consistir de uma fonte renovável de energia e contribuir para a mitigação das emissões de $\mathrm{CO}_{2}$, o biodiesel é mais facilmente degradado do que os derivados de petróleo, além de apresentar um baixo potencial tóxico (PRATES; PIEROBON; COSTA, 2007; PASQUALINO; MONTANÉ;
SALVADÓ, 2006; LAPINSKIENÉ; MARTINKUS; REBZDAITÉ, 2006; MARIANO, 2006)

Em 13 de janeiro de 2005, foi sancionada a Lei no 11.097, que permitiu a mistura de $2 \%$ de biodiesel ao diesel (B2) e estipulou prazo de 3 anos para a mistura se tornar obrigatória no território brasileiro. Em 2013, 8 anos após a promulgação da lei, o percentual obrigatório de mistura seria de 5\% (B5), o que foi antecipado para 2010.

A maior parte das pesquisas realizadas com o biodiesel está relacionada com o seu potencial energético, estabilidade térmica, 
condições ideais de armazenamento e níveis de emissões atmosféricas do combustível, além de desenvolvimento e cultivo de variedades (vegetais) com maior produtividade. Os trabalhos relativos aos reais impactos de misturas de óleos diesel e biodiesel nos corpos hídricos em caso de acidentes são bastante escassos (PRATES; PIEROBON; COSTA, 2007; DEMELLO et al., 2007).

Acidentes durante transporte por navios, caminhões ou dutos, e vazamentos em tanques de armazenamento de diesel — subterrâneos ou não - constituem problemas ambientais de grande impacto, causando poluição do solo e das águas, além de afetar toda a biota. A contaminação do solo pode atingir as águas subterrâneas, incluindo reservas já em uso como fontes de abastecimento para consumo humano (PEREIRA; MARQUES, 2009; MATER et al., 2007). Leme et al. (2012) observaram efeitos genotóxicos, mutagênicos e citotóxicos em águas contaminadas com biodiesel e suas misturas com diesel. Estudos realizados com vazamentos de misturas de biodiesel e diesel no mar (DEMELLO et al., 2007), mostraram que a presença dos óleos vegetais aumentou a dissolução e dispersão dos hidrocarbonetos do petróleo, em virtude da semelhança estrutural dos óleos vegetais com agentes tensoativos, agravando os impactos de contaminação em organismos aquático e bentônicos. O efeito da adição do biodiesel sobre a biodegradabilidade das misturas diesel/biodiesel não é bem estabelecido. Pasqualino, Montané e Salvadó (2006) e Zhang et al. (1998) indicaram efeito sinérgico positivo da adição do biodiesel ao diesel, pois os micro-organismos utilizam os ácidos graxos que compõem o biodiesel como fonte de energia para promover a degradação do diesel. Para frações contendo porções menores de biodiesel, o efeito sinérgico foi pouco significativo. Demello et al. (2007) apresentaram resultados divergentes, mostrando que a biodegradabilidade das misturas não seria favorecida pela presença do biodiesel. Conforme estes últimos estudos, as áreas poluídas com as misturas de biodiesel/diesel ou com diesel puro praticamente não apresentam diferenças quanto aos compostos presentes.

A remediação das áreas contaminadas in situ (no local, sem retirada do material contaminado) utiliza principalmente processos biológicos, fundamentados na ação de micro-organismos autóctones, e mais recentemente, processos de oxidação química fundamentados na ação de radical hidroxila (processos oxidativos avançados - POA). Os processos oxidativos têm sido aplicados também nos tratamentos on site (no local, após retirada da fração contaminada) ou ex situ (retirada da fração contaminada para tratamento em outro local) da água contaminada (NOGUEIRA et al., 2007; TIBURTIUS; PERALTA-ZAMORA; EMMEL, 2009).

Os POA têm recebido grande interesse no tratamento e pré-tratamento de compostos não biodegradáveis em águas, emissões atmosféricas e solos contaminados, pois podem converter a matéria orgânica em dióxido de carbono $\left(\mathrm{CO}_{2}\right)$ e água $\left(\mathrm{H}_{2} \mathrm{O}\right)$ ou, no caso de pré-tratamentos, tornar esta matéria biodegradável (ALOUI; KCHAOU; SAYADI, 2009; MORAIS; ZAMARA, 2005). Um dos POA mais promissores consiste na reação entre íon ferroso $\left(\mathrm{Fe}^{2+}\right) \mathrm{e}$ peróxido de hidrogênio $\left(\mathrm{H}_{2} \mathrm{O}_{2}\right)$ em meio ácido, conhecida como a "reação de Fenton", que gera os radicais hidroxila $(\bullet \mathrm{OH})$, de elevado potencial padrão de redução, com ataque pouco ou não seletivo às espécies presentes no meio reacional, em uma reação espontânea e que ocorre na ausência de luz (SILVA et al., 2005; DENG; ENGLEHARDT, 2006). Conforme Deng e Englehardt (2006), a dosagem do $\mathrm{H}_{2} \mathrm{O}_{2}$ depende da carga orgânica inicial do composto orgânico a ser tratado, expressa em termos da demanda química de oxigênio (DQO), estabelecendo uma dosagem ótima $\mathrm{DQO}_{0}: \mathrm{H}_{2} \mathrm{O}_{2}$ de 1:2. Este trabalho também aborda a relação ótima entre $\mathrm{H}_{2} \mathrm{O}_{2} \mathrm{e}$ $\mathrm{Fe}^{2+}$ necessária para maximizar a produção de $\cdot \mathrm{OH}$, que depende das características do composto orgânico, variando entre relações bem elevadas (11:1 a 35:1) e mais baixas (1,5:1 a 3:1). É importante controlar as concentrações de $\mathrm{Fe}^{2+} \mathrm{e}_{2} \mathrm{O}_{2}$, pois sua presença em excesso torna-se prejudicial, uma vez que podem capturar os radicais -OH (MORAIS; ZAMARA, 2005; MATER et al., 2007).

Embora haja registros na literatura de obtenção de constantes cinéticas para vários substratos orgânicos submetidos a tratamento por Fenton (PONTES et al., 2010; LOPEZ et al., 2003; RIOJA, 2010), não foram verificados estudos com Fenton empregando diesel ou biodiesel.

Neste trabalho foi estudada a aplicação do processo Fenton em águas contaminadas com misturas de diesel e biodiesel em diferentes proporções, com determinação das constantes cinéticas de degradação e avaliação da alteração de biodegradabilidade após o tratamento.

\section{Material e métodos}

\section{Ensaios de solubilidade}

As amostras utilizadas no trabalho foram preparadas em laboratório, pela mistura, em água destilada, de diesel/biodiesel em proporções pré-definidas. O estabelecimento das quantidades de diesel e biodiesel empregadas foi feito em duas etapas:

- solubilidade em 2 horas: foram preparadas 10 soluções de cada combustível testado, com concentrações variando de 10 a 100 g.L.-1. As soluções foram mantidas sob agitação a $150 \mathrm{rpm}$, em shaker (Quimis, modelo Q816M20), à temperatura ambiente, e ao final de duas horas, foram filtradas, após a separação de fases. As amostras filtradas foram submetidas à análise de carbono orgânico total (COT);

- solubilidade em 48 horas: foi preparada uma amostra de 80 g.L-1 para cada combustível; as amostras foram mantidas sob agitação em shaker durante 48 horas, com retirada de alíquotas ao longo do tempo, que foram filtradas e submetidas à análise de COT. 
Os ensaios foram realizados para dois tipos de biodiesel e um de óleo diesel, cujas características são apresentadas na Tabela 1. O biodiesel tipo 1 foi empregado nos ensaios de biodegradabilidade, e nos ensaios com reagente de Fenton foi utilizado o biodiesel tipo 2 .

\section{Preparo das amostras}

As amostras utilizadas nos ensaios de biodegradabilidade $\mathrm{e}$ Fenton foram preparadas a partir da mistura de $400 \mathrm{~g}$ de diesel/biodiesel em $5 \mathrm{~L}$ de água destilada. As misturas diesel/biodiesel recebem a denominação BX, em que $X$ é a percentagem, em massa, de biodiesel. As proporções empregadas foram B0, B25, B50, B75 e B100. As soluções foram mantidas sob agitação, a $150 \mathrm{rpm}$, em recipiente aberto, durante 48 horas. Após separação das fases, a fase aquosa foi filtrada e armazenada.

\section{Ensaios de biodegradabilidade}

Como os testes de Zahn-Wellens (OECD, 1992) são indicados para substratos solúveis, foram realizados ensaios utilizando o procedimento Zahn-Wellens modificado (NORR; MEINECKE; BRACKEMANN, 2000), uma adaptação para substâncias pouco solúveis, com o objetivo de comparar o comportamento das amostras filtradas e não filtradas. Estes ensaios foram realizados somente para o B100. Nos testes de biodegradabilidade subsequentes, foi utilizado o método Zahn-Wellens, empregando sempre amostras filtradas. Em todos os procedimentos foi utilizado como inóculo lodo de estação de tratamento de esgoto.

Foram preparados frascos de um litro contendo a mistura de óleos combustíveis, a solução de nutrientes minerais e a quantidade calculada de inóculo. O volume de inóculo foi calculado a partir da razão recomendada igual a 1:1, em termos de COT, entre as cargas orgânicas do próprio inóculo e do composto teste.

Foram também preparados dois testes paralelos, um contendo apenas o inóculo e os nutrientes minerais (teste branco) e o segundo contendo um composto de referência (glicose).

A biodegradabilidade dos compostos foi acompanhada ao longo do tempo até o teor de matéria orgânica, medido como COT, se manter constante.

Tabela 1 - Características dos óleos biodiesel e diesel.

\begin{tabular}{|c|c|c|}
\hline Biodiesel & & Diesel \\
\hline Tipo 1 & $\begin{array}{c}\text { Rota metílica; } 80 \% \text { soja e } 20 \% \\
\text { algodão }\end{array}$ & \multirow{2}{*}{$\begin{array}{l}\text { S500 tipo } A \text { - baixo } \\
\text { teor de enxofre }\end{array}$} \\
\hline Tipo 2 & $\begin{array}{c}\text { Rota metílica; } 95 \% \text { soja e 5\% } \\
\text { algodão }\end{array}$ & \\
\hline
\end{tabular}

\section{Testes Fenton}

Os experimentos foram conduzidos em agitadores Jar-test (PoliControl, modelo Floc Control II) de seis e três posições, utilizando dois litros de amostra a cada ensaio. Como reagentes utilizou-se sulfato ferroso heptahidratado $\left(\mathrm{FeSO}_{4} \cdot 7 \mathrm{H}_{2} \mathrm{O}\right)$ e o $\mathrm{H}_{2} \mathrm{O}_{2} 5 \%$. O ajuste do $\mathrm{pH}$ foi realizado utilizando-se soluções $0,1 \mathrm{~N}$ de ácido sulfúrico $\left(\mathrm{H}_{2} \mathrm{SO}_{4}\right)$ e $5 \%$ de hidróxido de sódio $(\mathrm{NaOH})$. Após o ajuste do $\mathrm{pH}$ inicial da amostra entre 3,5 e 4, iniciou-se a agitação (150 rpm) e foram adicionadas as quantidades pré-calculadas de $\mathrm{FeSO}_{4} \cdot 7 \mathrm{H}_{2} \mathrm{O}$, seguida da adição de $\mathrm{H}_{2} \mathrm{O}_{2}$. Adotou-se uma relação aproximada de $\mathrm{DQO}_{0}:\left[\mathrm{H}_{2} \mathrm{O}_{2}\right]:[\mathrm{Fe}]=1: 2: 2$, seguindo cálculos apresentados na literatura (DENG; ENGLEHARDT, 2006).

A reação foi acompanhada ao longo de uma hora por análises de COT, DQO e $\mathrm{H}_{2} \mathrm{O}_{2}$ residual. Nos primeiros 5 minutos de reação, foram retirados, a cada minuto, $10 \mathrm{~mL}$ de amostra para realização do estudo cinético de degradação da matéria orgânica em termos de DQO. As amostras retiradas foram neutralizadas com $\mathrm{NaOH}$ \% e, em seguida, foi adicionada enzima catalase para reagir com $\mathrm{O}_{2} \mathrm{O}_{2}$ residual. Todas as amostras analisadas foram previamente filtradas a vácuo utilizando membrana de fibra de vidro $45 \mathrm{~mm}$ (Sartorius) para remoção do ferro precipitado na etapa de neutralização.

\section{Técnicas analíticas}

Para preparo das amostras filtradas, foi utilizado papel filtro quantitativo universal de velocidade média (J. Prolab JP40).

A remoção da matéria orgânica nas diversas misturas diesel/ biodiesel foi avaliada através da evolução do $\mathrm{CO}_{2}$, do COT e da DQO. O COT foi analisado em um analisador de COT (Shimadzu, modelo $5000 \mathrm{~A}$ ), e a DQO pelo método colorimétrico, refluxo fechado (APHA; AWWA; WEF, 2005). A evolução de $\mathrm{CO}_{2}$ foi calculada a partir da absorção em hidróxido de bário, conforme procedimento Zahn-Wellens modificado (NORR; MEINECKE; BRACKEMANN, 2000).

A concentração de $\mathrm{H}_{2} \mathrm{O}_{2}$ residual nas amostras processadas foi determinada através do método iodométrico.

Foram realizadas análises da demanda bioquímica de oxigênio $\left(\mathrm{DBO}_{5}\right)$ das misturas dos combustíveis antes e após tratamento com Fenton, usando inóculo comercial (Gorduraklin ${ }^{\circledR}$, Bio-Brasil Limpeza Biológica Ltda.). O oxigênio dissolvido (OD) foi medido pelo método de Winkler modificado (APHA; AWWA; WEF, 2005).

Para interromper a reação de Fenton e eliminar da interferência do $\mathrm{H}_{2} \mathrm{O}_{2}$ residual nas análises de DQO, $\mathrm{DBO}_{5}$ e COT, a solução foi neutralizada e, em seguida, foi adicionada a enzima catalase, conforme procedimento descrito por Poole (2004). 


\section{Resultados e discussões}

\section{Ensaios de solubilidade}

A Figura 1 mostra os resultados das concentrações dos combustíveis na fase aquosa, em termos de COT, para diferentes quantidades de diesel e biodiesel do tipo 1 em $1 \mathrm{~L}$ de água deionizada. Verificou-se que a partir de $80 \mathrm{mg} . \mathrm{L}^{-1}$ para o diesel e $70 \mathrm{mg} . \mathrm{L}^{-1}$ para o biodiesel, praticamente não houve variação da solubilidade (em termos de COT), indicando a saturação da solução.

Fixando a concentração dos óleos em 80 g. $\mathrm{L}^{-1}$ em termos de COT e estendendo-se o tempo de agitação, observou-se que, para o biodiesel, há ainda uma fração importante que pode ser solubilizada após 2 h de contato (Figura 2). O tempo de agitação tem uma maior influência sobre a solubilidade do biodiesel, enquanto para o diesel, este se mostrou menos importante. Segundo Shah et al. (2010), os valores de solubilidade mais elevados para o biodiesel devem-se ao fato do biodiesel apresentar certa polaridade e portanto, afinidade em se ligar com a água. Observa-se também que os valores de solubilidade de biodiesel variam para os diferentes tipos, confirmando os resultados apresentados por Hollebone

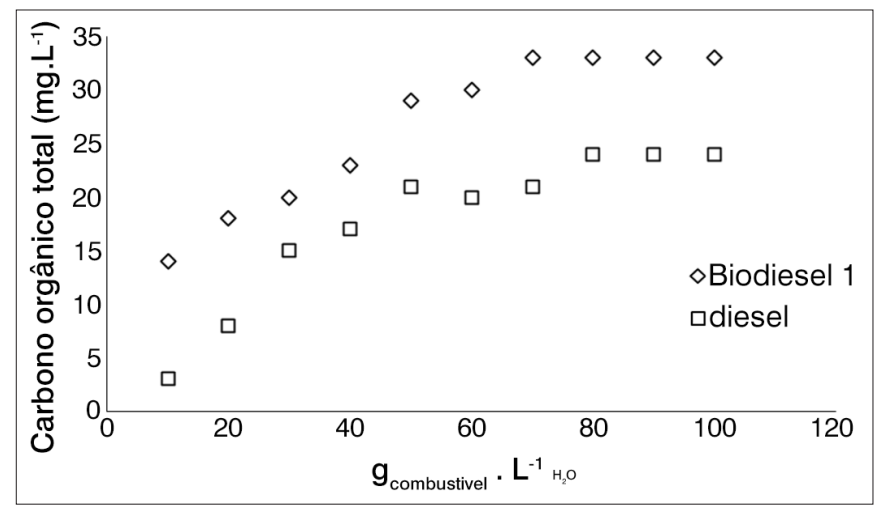

Figura 1 - Solubilidade dos óleos combustíveis, em termos de carbono orgânico total, variando a quantidade dos óleos adicionada.

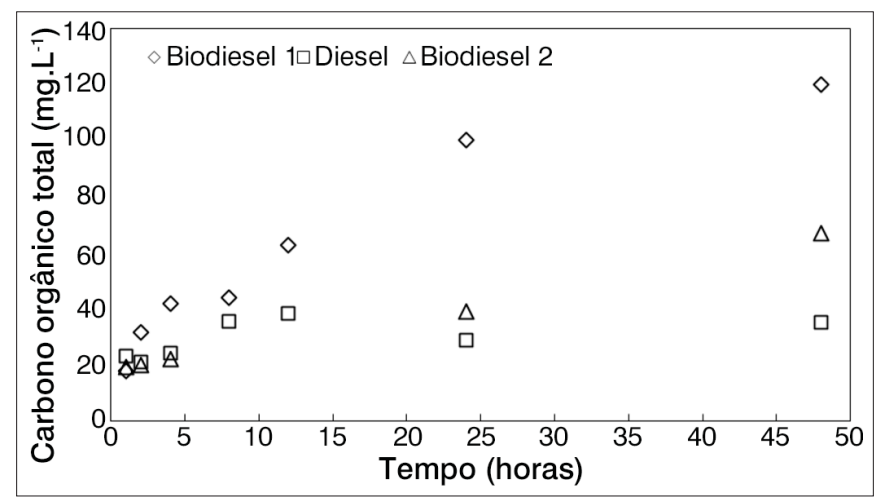

Figura 2 - Solubilidade, em termos de carbono orgânico total, de amostras contendo $80 \mathrm{~g}$ de óleo combustível em $1 \mathrm{~L}$ de água, variando o tempo de agitação. et al. (2008), que relatam variações na solubilidade do biodiesel, mesmo sendo proveniente da mesma matéria prima ou produzidos pela mesma rota.

Face aos resultados de solubilidade, foi estabelecido que as amostras para a pesquisa seriam preparadas pela adição de $80 \mathrm{~g}$ dos combustíveis em 1 litro de $\mathrm{H}_{2} \mathrm{O}$, mantidos sob agitação por 48 horas e filtradas após separação da fase aquosa. As amostras de biodiesel tipo 1 foram empregadas nos testes de biodegradabilidade, e o tipo 2 para o processo Fenton, incluindo a avaliação da biodegradabilidade antes e após oxidação.

\section{Ensaios de biodegradabilidade}

A Figura 3 mostra os resultados dos testes de biodegradabilidade para B100, filtrado e não filtrado, e glicose, utilizando o procedimento Zahn Wellens modificado.

Os testes de B100 filtrado e não filtrado apresentaram resultados semelhantes. Este comportamento já era esperado levando-se em consideração que os micro-organismos somente decompõem a fração solúvel dos componentes (Lapinskiené; Martinkus; Rebzdaité, 2006; DEMELLO et al., 2007; ZHANG et al., 1998).

Analisando os dados apresentados na Figura 3, verificou-se, para os combustíveis, que houve uma fase de adaptação dos micro-organismos nos três primeiros dias, com baixa degradabilidade. A degradação dos biocombustíveis atingiu valores de $60 \%$ no terceiro dia de teste, estabilizando em valores superiores a 70\% após o décimo dia. Os resultados confirmaram que o biodiesel é facilmente degradado (PASQUALINO; MONTANÉ; SALVADÓ, 2006; LAPINSKIENÉ; MARTINKUS; REBZDAITÉ, 2006; MARIANO, 2006) e mostram que não há diferença de biodegradabilidade quando se trabalha com o biodiesel filtrado e não filtrado. O fato do biocombustível não alcançar maiores percentagens de

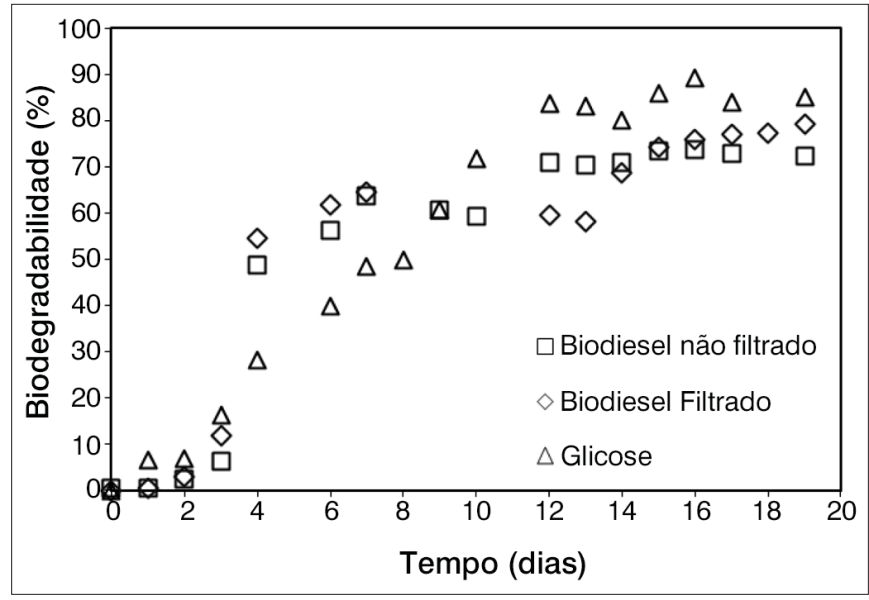

Figura 3 - Curva de biodegradabilidade para biodiesel não filtrado, biodiesel filtrado e glicose, obtida através do método Zahn-Wellens modificado. 
biodegradabilidade pode estar relacionado aos baixos índices de oxigênio dissolvido constatados no frasco respirométrico e às próprias limitações do método (ZHANG et al.,1998). A baixa degradabilidade da glicose observada nos primeiros dias pode ser explicada pelo inóculo utilizado (lodo de ETE), aclimatado a material orgânico de natureza diversa e mais complexo que a glicose; o mesmo comportamento foi observado e relatado por Amaral (2009).

O método de Zahn-Wellens modificado mostrou-se bastante trabalhoso em virtude das trocas contínuas das soluções de hidróxido de bário $\left(\mathrm{Ba}(\mathrm{OH})_{2}\right)$ nas colunas de absorção, do desgaste das bombas peristálticas, bem como da alimentação descontínua de oxigênio. Uma vez que no processo Fenton homogêneo e nos ensaios de biodegradabilidade a fração solúvel dos óleos é o substrato, foi estabelecido que em todos os testes subsequentes seriam empregadas sempre as amostras filtradas dos óleos e suas misturas, usando o método de Zahn-Wellens sem modificações.

A Figura 4 mostra os resultados dos ensaios de biodegradabilidade para cinco diferentes misturas biodiesel-diesel. Pode-se verificar que o B100 atingiu uma biodegradabilidade de 100\% em 10 dias de teste, enquanto para o B0 este valor foi de cerca de 30\%, em 28 dias de teste. A biodegradabilidade final do B0 e B5 foi similar, mostrando que para amostras contendo pequenas porções de biodiesel, a degradação biológica praticamente não foi afetada. Entretanto, a biodegradabilidade das misturas aumentou com o acréscimo do biodiesel, sendo que esse aumento se tornou mais evidente a partir da mistura B75. Pode-se também verificar que o B100 e B75 mostraram elevada velocidade inicial, atingindo valores entre 90 e $60 \%$, respectivamente, em 2 dias de testes, seguidas por uma segunda fase em que a decomposição biológica é reduzida. As curvas do B0 e B5 também mostraram que a maior parte da biodegradabilidade ocorreu nos dois primeiros dias, apresentando maior velocidade de degradação.

Os valores da biodegradabilidade final obtidos foram semelhantes àqueles obtidos em trabalho realizado por Pasqualino; Montané; Salvadó (2006)

\section{Testes Fenton}

Nos primeiros ensaios cinéticos, utilizou-se uma relação aproximada de $\mathrm{DQO}_{0}:\left[\mathrm{H}_{2} \mathrm{O}_{2}\right]:\left[\mathrm{Fe}^{2+}\right]=1: 2: 2$, seguindo cálculos apresentados na literatura (DENG; ENGLEHARDT, 2006). Entretanto, os resultados mostraram que as reações foram muito rápidas, e que se estabilizaram em tempos pequenos, em um período inferior a um minuto. Em virtude da baixa concentração de carbono dissolvido na amostra após o emprego do sistema Fenton (<20 mg. $\mathrm{L}^{-1}$ COT), não foi possível realizar o ensaio de biodegradabilidade pós-Fenton. A avaliação da biodegradabilidade das amostras foi feita pela relação $\mathrm{DBO}_{5} / \mathrm{DQO}$ da amostra antes e após tratamento com Fenton. Na Figura 5 pode-se observar um aumento da relação $\mathrm{DBO}_{5} / \mathrm{DQO}$ na amostra tratada com Fenton, variando este incremento entre 0,3 e 0,5, o que representa um aumento de até $150 \%$ na biodegradabilidade

Outros ensaios foram realizados para estabelecer as condições ótimas para os ensaios cinéticos, reduzindo-se a relação $\mathrm{DQO}_{0}: \mathrm{H}_{2} \mathrm{O}_{2}$. Foram definidas as relações $\mathrm{DQO}_{0}: \mathrm{H}_{2} \mathrm{O}_{2}: \mathrm{Fe}^{2+}$ de $2: 1: 1$ para $\mathrm{B} 0 ; 1: 1: 1$ para B25, B50 e B75. Foram coletadas amostras a cada minuto, nos cinco minutos iniciais, para análise de DQO. Os resultados dos testes estão apresentados na Figura 6.

Mesmo empregando uma quantidade reduzida de $\mathrm{H}_{2} \mathrm{O}_{2}$, comparada com aquela sugerida pela literatura (DENG; ENGLEHARDT, 2006) e adotada nos testes iniciais, observou-se que a adição de biodiesel favorece a oxidação química. Para o B25, a remoção foi da ordem de $80 \%$, e superior a $80 \%$ para B50 e B75, sendo que, para esta última mistura, a redução alcançou cerca de $90 \%$.

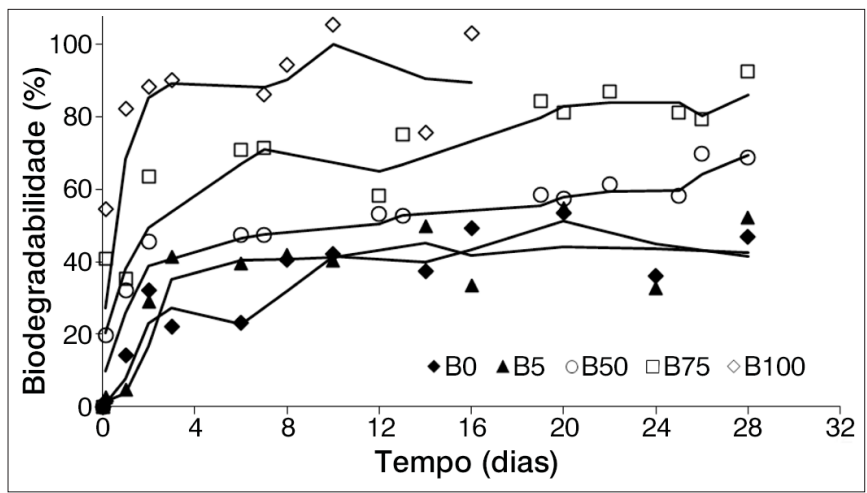

Figura 4 - Biodegradabilidade das misturas dos combustíveis obtida através do método Zahn-Wellens.

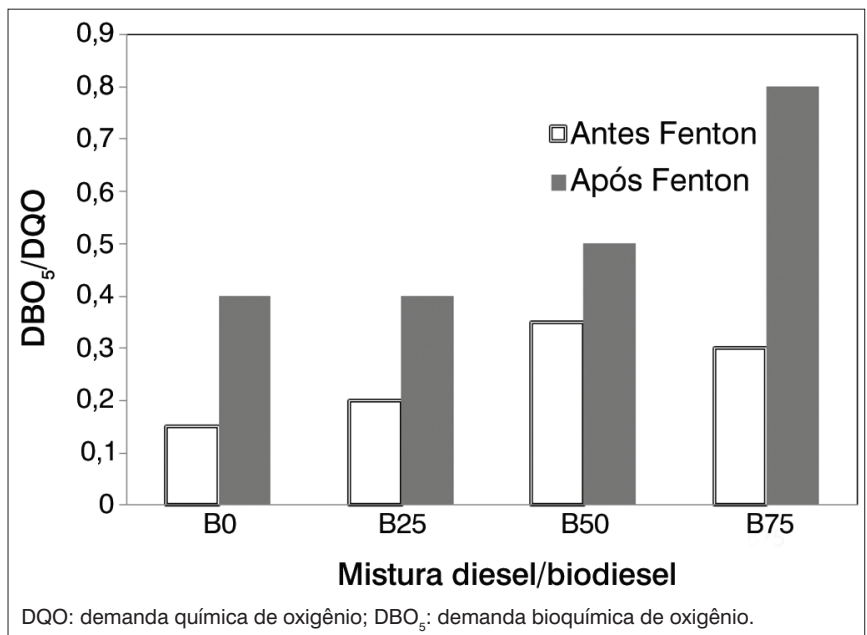

Figura 5 - Biodegradabilidade, em termos da relação demanda bioquímica de oxigênio/demanda química de oxigênio ( $\left.\mathrm{DBO}_{5} / \mathrm{DQO}\right)$, para as misturas diesel/biodiesel antes e após tratamento por processo Fenton. Condições operacionais: $\mathrm{DQO}: \mathrm{H}_{2} \mathrm{O}_{2}: \mathrm{Fe}^{2+}$ de 0,5:1:1 
Para a determinação das constantes cinéticas, foram utilizados os dados dos cinco primeiros minutos de reação. A representação do inverso da DQO (1/DQO) em função do tempo possibilitou melhor ajuste dos dados, conforme mostrado na Figura 7.

Para todas as misturas estudadas, os coeficientes de correlação apresentaram valores superiores a 0,9, exceto para o B0.

Portanto, considerou-se que a cinética de remoção da DQO por Fenton foi de pseudo-segunda ordem (uma vez que foi considerada apenas a variação da DQO) para os substratos estudados, exceto para B0. Os valores das constantes cinéticas (Tabela 2) são da mesma ordem de magnitude, indicando que todas as amostras apresentaram reatividade bastante semelhante com relação ao ataque da $\bullet \mathrm{OH}$. Houve um aumento da velocidade quando a quantidade de biodiesel na mistura foi elevada de 25 para $50 \%$;

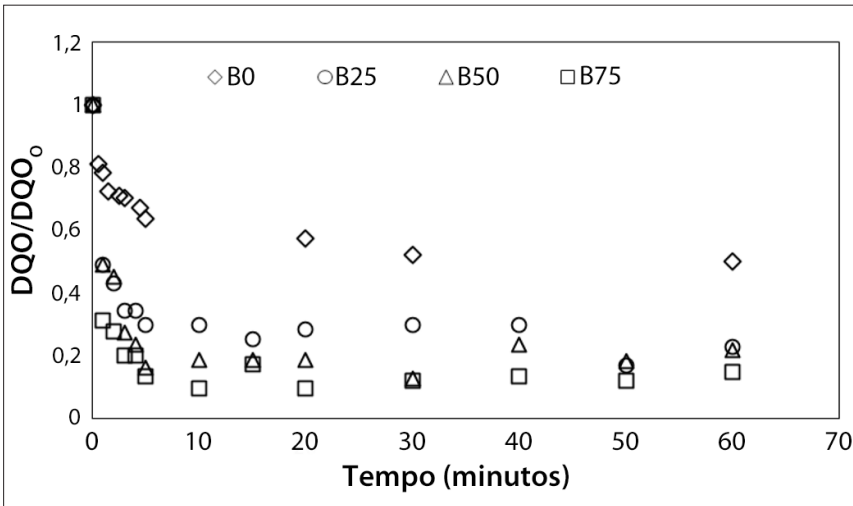

DQO: demanda química de oxigênio; $\mathrm{DQO}_{0}$ : demanda química de oxigênio inicial das amostras

Figura 6 - Variação da demanda química de oxigênio ao longo de 60 minutos. Condições experimentais $\mathrm{DQO}_{0}:\left[\mathrm{H}_{2} \mathrm{O}_{2}\right]:\left[\mathrm{Fe}^{2+}\right]=1: 1: 1$ para $\mathrm{B} 25$, $\mathrm{B} 50$ e B75; $\mathrm{DQO}_{0}:\left[\mathrm{H}_{0} \mathrm{O}_{2}\right]:\left[\mathrm{Fe}^{2+}\right]=2: 1: 1$ para $\mathrm{BO} ; \mathrm{pHO}=3,6 ; 150 \mathrm{rpm}$.

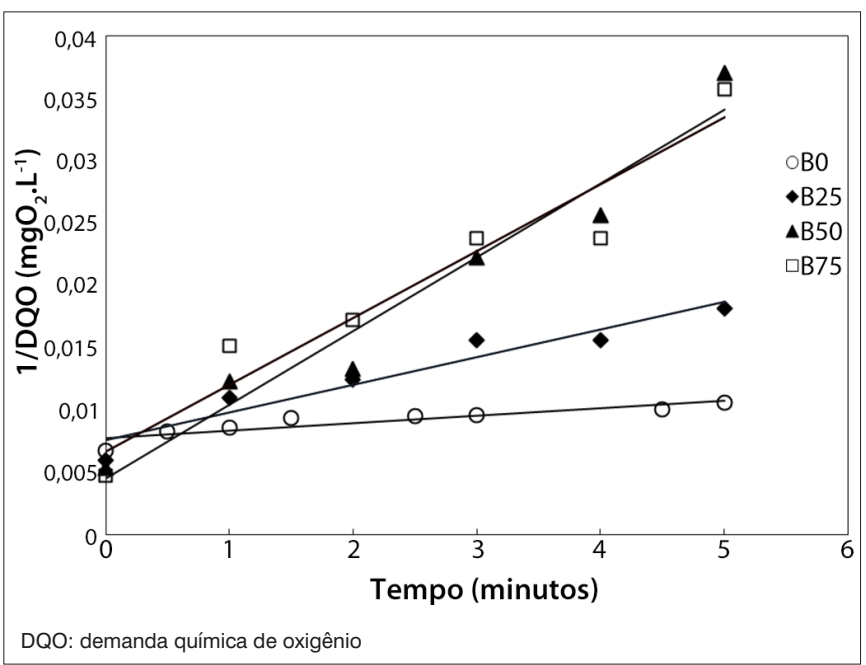

Figura 7 - Cinética de segunda ordem para a reação Fenton. Condições experimentais $\mathrm{DQO}_{0}:\left[\mathrm{H}_{2} \mathrm{O}_{2}\right]:\left[\mathrm{Fe}^{2+}\right]=1: 1: 1$ para $\mathrm{B} 25$, B50 e $\mathrm{B} 75$; $\mathrm{DQO}_{0}:\left[\mathrm{H}_{2} \mathrm{O}_{2}\right]:\left[\mathrm{Fe}^{2+}\right]=2: 1: 1$ para $\mathrm{BO} ; \mathrm{pHO}=3,6 ; 150 \mathrm{rpm}$.
Tabela 2 - Constantes cinéticas para reação de segunda ordem e coeficientes de correlação.

\begin{tabular}{lcc} 
Amostra & $\mathrm{k}\left(\mathrm{mg}^{\mathrm{L}-1} \cdot \mathrm{min}^{-1}\right)$ & $\mathrm{R}^{2}$ \\
\hline B0 & $6,0 \cdot 10^{-4}$ & 0,8197 \\
B25 & $2,2 \cdot 10^{-3}$ & 0,9259 \\
B50 & $5,9 \cdot 10^{-3}$ & 0,9554 \\
B75 & $5,4 \cdot 10^{-3}$ & 0,9284
\end{tabular}

$\mathrm{R}^{2}$ : coeficiente de correlação; BX: os números especificam o percentual em massa de biodiesel na mistura.

Tabela 3 - Constantes cinéticas verificadas na literatura sobre estudos de degradação por Fenton de compostos orgânicos refratários.

\begin{tabular}{lccc} 
Referência & Modelo cinético & Composto & $\begin{array}{c}\text { Constante } \\
\text { cinética } \\
\left(\mathrm{mg}^{-1} \cdot \mathrm{min}^{-1}\right)\end{array}$ \\
$\begin{array}{l}\text { Pontes et al. } \\
(2010)\end{array}$ & $\begin{array}{c}\text { Pseudo-segunda } \\
\text { ordem }\end{array}$ & Fenol & $8,5 \cdot 10^{6}$ \\
$\begin{array}{l}\text { Lopez et al. } \\
\text { (2003) }\end{array}$ & $\begin{array}{c}\text { Pseudo-segunda } \\
\text { ordem }\end{array}$ & $\begin{array}{c}\text { Intermediário } \\
\text { farmacêutico }\end{array}$ & 3,78 \\
$\begin{array}{l}\text { Rioja et al. } \\
(2010)\end{array}$ & $\begin{array}{c}\text { Pseudo-segunda } \\
\text { ordem }\end{array}$ & Surfactante & $1,2.10^{-4}$ \\
\hline
\end{tabular}

entretanto, ela manteve-se praticamente constante quando a proporção variou de 50 para $75 \%$.

Estudos verificados na literatura sobre degradação de fenol por Fenton (PONTES et al., 2010), degradação de intermediários farmacêuticos em soluções aquosas utilizando radiação ultravioleta (UV) e UV/ $\mathrm{H}_{2} \mathrm{O}$ (LOPEZ et al., 2003) e degradação de surfactantes utilizando Fenton (RIOJA, 2010) também apresentaram um modelo cinético de segunda ordem com relação ao substrato. Os valores das constantes variam para cada substrato, conforme mostrado na Tabela 3.

\section{Conclusões}

A biodegradabilidade do diesel não se alterou por pequenos acréscimos de biodiesel, obtendo se para B0 e para B5 30\% de redução da DQO após 28 dias de ensaio. A alteração da biodegradabilidade da mistura biodiesel/diesel somente foi significativa a partir do B75, para o qual se verificou uma remoção de $80 \%$ da matéria orgânica após 28 dias.

O processo Fenton proporcionou uma remoção da matéria orgânica superior a $80 \%$ para todas as misturas estudadas, exceto para o B0, que foi de 50\%. A remoção de carga orgânica pelo processo Fenton é bastante elevada mesmo com a utilização de metade da quantidade de $\mathrm{H}_{2} \mathrm{O}_{2}$ indicada na literatura.

Após tratamento por processo Fenton, as misturas diesel/biodiesel apresentaram aumento de biodegradabilidade de até 150\%, o que permite uma melhor na eficiência e redução dos custos em um posterior tratamento biológico. 
O ajuste cinético da reação de oxidação das misturas dos combustíveis pelo processo Fenton mostrou que a redução da DQO pode ser descrita por um modelo de pseudo-segunda ordem.

\section{Agradecimentos}

À Fundação de Amparo à Pesquisa do Estado de Minas Gerais (FAPEMIG) — Processos TEC PPM-00011/10 e TEC APQ-03944-10.

\section{Referências}

ALOUI, F.; KCHAOU, S.; SAYADI, S. (2009) Physicochemical treatments of anionic surfactants wastewater: effect on aerobic biodegradability. Journal of Hazardous Materials, n. 164, p. 353-359.

AMARAL, M.C.S. (2009) Tratamento de efluente de branqueamento de polpa celulósica empregando sistema de microfiltração conjugado com biorreator de membrana. Tese (Doutorado em Saneamento, Meio Ambiente e Recursos Hídricos) - Escola de Engenharia, Universidade Federal de Minas Gerais, Belo Horizonte.

AMERICAN PUBLIC HEALTH ASSOCIATION (APHA); AMERICAN WATER WORKS ASSOCIATION (AWWA); WATER ENVIRONMENT FEDERATION (WEF). Standard Methods for the Examination of Water and Wastewater. 21st ed. Washington: APHA; AWWA; WEF, 2005.

DEMELLO J.A.; CARMICHAEL C.A.; PEACOCK E.E.; NELSON R.K.; AREY J.S.; REDDY, C.M. (2007) Biodegradation and environmental behavior of biodiesel mixtures in the sea: an initial study. Marine Pollution Bulletin, n. 54, p. 894-904.

DENG, Y.; ENGLEHARDT, J.D. (2006) Treatment of landfill leachate by the Fenton process. Water Research, v. 40, n. 20, p. 3683-3694

HOLLEBONE, B.P.; FIELDHOUSE, B.; LANDRIANULT, M.; DOE, K.; JACHMAN, P. (2008) Aqueous solubility, dispersibility and toxicity of biodiesels. In: International Oil Spill Conference, Portland, Oregon.

LAPINSKIENÉ A.; MARTINKUS P.; REBZDAITÉ V. (2006) Eco-toxicological Studies of diesel and biodiesel fuels in aerated soil. Environment Pollution, v.142, p.432-437.

LEME, D.M.; GRUMMT, T.; OLIVEIRA, D.P.; SEHR, A.; RENZ, S.; REINE, S.; FERRAZ, E.R.A.; MARCHI, M.R.R.; MACHADO, M.C.; ZOCOLO, G.J.; MARIN-MORALES, M.A. (2012) Genocity assessment of water soluble fractions of biodiesel and its diesel blends using Salmonella assay and the in vitro MicroFlow kit (Litron) assay. Chemosphere, v. 86, n. 5, p. 512-520.

LOPEZ, A.; BOZZI, A.; MASCOLO, G.; KIWI, J. (2003) Kinetic investigation on UV and $\mathrm{UV} / \mathrm{H}_{2} \mathrm{O}_{2}$ degradations of pharmaceutical intermediates in aqueous solution. Journal of Photochemistry and Photobiology A: Chemistry, v. 156, p.121-126.

Mariano, A.P. (2006) Avaliação do potencial de biorremediação de solos e de águas subterrâneas contaminados com óleo diesel. Tese (Doutorado em Geociência e Meio Ambiente) - Universidade Estadual Paulista, Rio Claro.
MATER, L.; ROSA, E.V.C.; BERTO, J., CORRÊA, A.X.R.; SCHWINGEL, P.R.; RADETSKI, C.M. (2007) A simple methodology to evaluate influence of $\mathrm{H}_{2} \mathrm{O}_{2}$ and $\mathrm{Fe}^{2+}$ concentrations on the mineralization and biodegradability of organic compounds in water and soil contaminates with crude petroleum. Journal of Hazardous Materials, v.149, n. 2, p. 379-386.

MORAIS, J.L.; ZAMORA, P.P. (2005) Use of advanced oxidation processes to improve the biodegradability of mature landfill leachates. Journal of Hazardous Materials B, n. 123, p. 181-186.

NOGUEIRA, R.F.P.; TROVÓ, A.G.; SILVA, M.R.A.; VILLA, R.D. (2007) Degradação de benzeno, tolueno e xilenos em águas contaminadas por gasolina, utilizando-se processos foto-fenton. Quimica Nova, v. 30, n. 2 , p. $400-408$

NORR, C.; MEINECKE, S.; BRACKEMANN, H. (2000) Modification of the Zahn-Wellens test: determination of the biodegradability of poorly soluble adsorbing and volatile substances by measurement of oxygen consumption and carbon dioxide production. Chemosphere, v. 44 , n. 4, p. 553-559.

ORGANISATION FOR ECONOMIC CO-OPERATION AND DEVELOPMENT (OECD). (1992) Guidelines for testing of chemicals. Zahn-Wellens/EMPA Test adopted by the Council on $17^{\text {th }}$ July 1992.

PASQUALINO J.C.; MONTANÉ D.; SALVADÓ J. (2006) Synergic effects of biodiesel in the biodegradability of fossil-diesel fuels. Biomass and Bioenergy, v. 30, n. 10, p.894-879.

PEREIRA, C.A.; MARQUES, M.R.C. (2009) Avaliação da potencialidade de processos pseudo-fenton para remediação de solos contaminados por diesel. Química Nova, v. 32, n. 8, p. 2200-2202.

PONTES, R.F.F.; MORAES, J.E.F.; MACHULEK A.; PINTO, J.M. (2010) A mechanistic kinetic model for phenol degradation by the Fenton process. Journal of Hazardous Materials, v. 176, n. 1-3, p. 402-413

POOLE, A. (2004) Treatment of biorefractory organic compounds in wool scour effluent by hidroxyl radical oxidation. Water Research, v. 38, n. 1415, p. 3458-3464.

PRATES C.P.; PIEROBON E.C.; COSTA R.C. (2007) Formação do mercado de biodiesel no Brasil. BNDES Setorial, n. 25, p. 39-64.

RIOJA, J.C.C. (2010) Uso do Resíduo Siderúrgico na adsorção e perocisdação catalítica de surfactantes aniônicos. 91p. Dissertação (Mestrado em Saneamento, Meio Ambiente e Recursos Hídricos) - Escola de Engenharia, Universidade Federal de Minas Gerais, Belo Horizonte. 
SHAH, P.; WEE, C.; WHITE, J.M.; SANFORD, S.; MEIER, G. FIELDHOUSE, B.; LANDRIANULT, M.; DOE, K.; JACHMAN, P. (2010) Experimental determination and thermodynamic modelling of water content in biodiesel-diesel blends. Renewable Energy Group, Inc.

SILVA, D.N.; ZAGATTO, P.J.P.; GUARDANI, R.; NASCIMENTO, C.A.O. (2005) Remediation of polluted soils contaminated with linear alkylbenzenes using Fenton's reagent. Brazilian Archives of Biology and Technology, v.48, p. 257-265.
TIBURTIUS, E.R.L.; PERALTA-ZAMORA, P.; EMMEL, A. (2009) Degradação de benzeno, tolueno e xilenos em águas contaminadas por gasolina, utilizando-se processos foto-Fenton. Química Nova, v. 32 n. 8, p. 2058-2063.

ZHANG X.; PETERSON C.; REECE D.; HAWS R.; MOLLER, G. (1998) Biodegradability of biodiesel in the aquatic environment. Transactions of the ASAE, v. 41, n. 5, p. 1423-1430. 